\title{
Abiotrophia defectiva: briefs comments of an etiological agent to consider in infections with conventional microbiological culture-negative
}

\section{Background}

Abiotrophia is a separate genus of Gram-positive cocci formerly classified as nutritionally variant streptococci (NVS). These microorganisms' forms part of the human oropharyngeal, gastrointestinal \& urogenital habitual microbiota, and cause $1 \%-2 \%$ of all infective endocarditis (IE), with the particular characteristic than these bacteria do not usually grow on conventional blood agar plates (culture-negative IE). Therefore, Abiotrophia endocarditis is classified into the category of culture-negative endocarditis, and their suspected implication should be informed to the microbiology laboratory to implement the recovery and isolation techniques to obtain the final identification of it, because the NVS are fastidious Gram-positive bacteria, requiring either L-cysteine- or pyridoxalsupplemented medium to support growth. We advised the physicians in culture-negative pathologies (besides IE) to think outside the box, and consider Abiotrophia participation in some infections without an etiological agent able to be identified, as we demonstrated in 2 of the 5 patients that we described. Is important to keep in mind this agent because their specific antimicrobial therapy could be failed in some cases, and the continued surveillance is needed to prevent the significant morbidity and mortality associated with this infection.

\section{Goal}

To briefly describe 5 isolates of $A$. defectiva in clinical samples of 5 patients with previous negative cultures ( 3 with infective endocarditis -IE-, 1 with pulmonary granulomatous disease \& 1 with chronic pungent vaginal discharge), received at the Medical Microbiology Unit Laboratory in the year 2010.

\section{Material \& methods}

In 2010, five patients' samples were received and processed for culture and isolations of aerobic \& anaerobic bacteria, fungus and mycobacterial agents (slow and fast-growing) according to the physicians' request. The stains of them were performed too. All the primary cultures were negative. After the suggestion to perform the investigation of unfrequently \& fastidious microorganisms', new samples arrive to our medical microbiology unit facility as follow:

I. 6 blood cultures (by duplicate) of 3 patients with clinical, echocardiographic and microbiological diagnosis of culturenegative IE.

II. 4 pleural fluid samples from a patient with suspected infectious granulomatosis, with fungal and mycobacterial culture negatives.

III. 2 samples of cervix-vaginal swab of patient with pungent vaginal discharge, negative for demonstration of specific vaginitis agents and bacterial vaginosis

\section{Results}

All samples were processing following CLSI recommendations Blood cultures and the rest of the samples were incubated and shows growth in liquid media (turbidity at 24-36 hours) with a Gram stain
Volume 7 Issue 2 - 2019

\author{
Marcel Marcano-Lozada, ${ }^{1,2}$ Silvia Molero- \\ Leon $^{3}$ \\ 'Department of Microbiology, Universidad Central de Venezuela, \\ Venezuela \\ ${ }^{2}$ Medical Microbiology Unit, Angios Vascular Center \& Wound \\ Clinic, Venezuela \\ ${ }^{3}$ Internal Medicine Specialist, Angios Vascular Center \& Wound \\ Clinic,Venezuela
}

\begin{abstract}
Correspondence: Marcel Marcano-Lozada,Aggregated Professor Microbiology Department,"J.M.Vargas" School of Medicine, Universidad Central de Venezuela, Caracas, Venezuela, Tel+506-57I905I6, Email marcelmarcano@gmail.com
\end{abstract}

Received: March 13, 2019 | Published: May 10, 2019

that reports "Gram-positive cocci in short chains", but with a negative subculture in the blood agar plates, a positive subculture in the enriched media (Chocolate agar incubated at $35^{\circ} \mathrm{C}$ in a humidified atmosphere with $5 \% \mathrm{CO}_{2}$ ) and the blood agar plate with a $S$. aureus stria (Satellite growth at the sides of the stria, at hemolysis zone). The growth of satellites bright white microcolonies, with alpha hemolysis and a Gram stain that shows Gram-positive cocci, in short chains \& pairs. The biochemical tests results were: Catalase negative, PYR (L-Pirrolidonil-arilamidase) positive, Bilis-Esculin negative, and lactose \& trehalose fermentation positive; all of it leading to the identification of $A$. defectiva as the causative agent of the diseases in the five patients.

\section{Discussion}

Lack of isolation in all previous cultures could be addressed to the no consideration of "fastidious" or demanding agents, and it was a primary diagnostic failure from the physician and the microbiology laboratory has the obligation to advise the need to expand the search to this kind of agents. So, if necessary, the two-ways communication between the clinic setting and the laboratory setting to improve the response time and perform accurate diagnosis that improves patients' treatment and recovery. The susceptibility test for Broth Micro dilution following recommendations of the Document M45-A2 CLSI (2010) demonstrating susceptibility to all tested antimicrobials (penicillin, oxacillin, ceftriaxone, vancomycin, clindamycin, linezolid, levofloxacin and gentamycin), and all patients have received at least 2 of this antimicrobial agents for the treatment of their diseases, but without any improvement signs, so, is time to consider the biofilmproducing strains as a factor to therapeutic failure?, but, all the isolated strains were negative for biofilm-production! So, a deep review of the antibiotic treatment record reveals that maybe the treatment times were responsible for the lack of improvement, because the recommended treatment duration for the Abiotrophia isolates is prolonged and the antimicrobial agents' combination is a great advice not only for IE. 
All the patients show improvement of its conditions with clinical and microbiological cure demonstrated at the end of a prolonged (3-6 weeks) and combined antimicrobial therapy. Is necessary the adequate determination of gender and species in order to optimize therapeutic regimens, and follow the antimicrobial susceptibility tests results, including new and powerful antimicrobials like daptomycin.

\section{Conclusion}

In cases where there is clinical suspicion of infection by species of Abiotrophia sp. and with conventional negative cultures, it is necessary to use supplemented liquid media or solids media enriched with pyridoxine and / or chocolate agar or use the technique of detection by satellite growth with a $S$. aureus strain to achieve the approximation to the microbiological diagnosis of this fastidious bacteria. ${ }^{1-5}$

\section{Acknowledgments}

None.

\section{Conflicts of interest}

Authors declare that there are no conflicts of interest.

\section{References}

1. Ruoff K. Aerococcus, Abiotrophia, and other infrequently isolated aerobic catalase-negative, grampositive cocci. In: Murray PR, Baron EJ, Editors. Manual of Clinical Microbiology. 8th edn. Washington. ASM Press; 2003:434-44.

2. Kawamura Y, Hou X, Sultana F, et al. Transfer of Streptococcus adjacens and Streptococcus defectivus to Abiotrophia gen. nov. as Abiotrophia adiacens comb. nov. and Abiotrophia defectiva comb. nov., respectively. Int J Syst Bacterial. 1995;45:798-803.

3. Roggenkamp A, Abele-Horn M, Trebesius K, et al. Abiotrophia elegans sp. nov., a possible pathogen in patients with culture negative endocarditis. J Clin Microbiol. 1998;36:100-104.

4. Marcano L MJ, Landaeta M JM. Endocarditis infecciosa. VITAE. $2004 ; 19$.

5. CLSI Methods for Antimicrobial Dilution and Disk Susceptibility Testing of Infrequently Isolated or Fastidious Bacteria. 2nd edn. Clinical and Laboratory Standards Institute; 2010. 